\title{
Relative mass distributions of neutron-rich thermally fissile nuclei within statistical model
}

\author{
Bharat Kumar ${ }^{1,4}{ }^{*}$ M.T. Senthil Kannan ${ }^{2}$, M. Balasubramaniam² , B. K. Agrawal ${ }^{3,4}$, and S. K. Patra ${ }^{1,4}$ \\ ${ }^{1}$ Institute of Physics, Sachivalaya Marg, Bhubaneswar - 751005, India. \\ ${ }^{2}$ Department of Physics, Bharathiar University, Coimbatore - 641046, India. \\ ${ }^{3}$ Saha Institute of Nuclear Physics, 1/AF, Bidhannagar, Kolkata - 700064, India. and \\ ${ }^{4}$ Homi Bhabha National Institute, Anushakti Nagar, Mumbai - 400094, India.
}

(Dated: October 2, 2018)

\begin{abstract}
We study the binary mass distribution for the recently predicted thermally fissile neutron-rich uranium and thorium nuclei using statistical model. The level density parameters needed for the study are evaluated from the excitation energies of temperature dependent relativistic mean field formalism. The excitation energy and the level density parameter for a given temperature are employed in the convolution integral method to obtain the probability of the particular fragmentation. As representative cases, we present the results for the binary yield of ${ }^{250} \mathrm{U}$ and ${ }^{254} \mathrm{Th}$. The relative yields are presented for three different temperatures $T=1,2$ and $3 \mathrm{MeV}$.

PACS numbers: 25.85.-w, 21.10.Ma, 21.10.Pc, 24.75.+i
\end{abstract}

\section{INTRODUCTION}

Fission phenomenon is one of the most interesting subject in the field of nuclear physics. To study the fission properties, a large number of models have been proposed. The fissioning of a nucleus is successfully explained by the liquid drop model and the semi-empirical mass formula is the best and simple oldest tool to get a rough estimation of the energy released in a fission process. The pioneering work of Vautherin and Brink [1], who has applied the Skyrme interaction in a selfconsistent method for the calculation of ground state properties of finite nuclei opened a new dimension in the quantitative estimation of nuclear properties. Subsequently, the HartreeFock and time dependent Hartree-Fock formalisms [2] are also implemented to study the properties of fission. Most recently, the microscopic relativistic mean field approximation, which is another successful theory in nuclear physics is also used for the study of nuclear fission [3].

From last few decades, the availability of neutron rich nuclei in various laboratories across the globe opened up new research in the field of nuclear physics, because of their exotic decay properties. The effort for the synthesis of superheavy nuclei in the laboratories like, Dubna (Russia), GSI (Germany), RIKEN (Japan) and BNL (USA) is also quite remarkable. Due to all these, the periodic table is extended till date upto atomic number $Z=118$ [4]. The decay modes of these superheavy nuclei are very different than the usual modes. Mostly, we understand that, a neutron rich nucleus has a large number of neutron than the light or medium mass region of the periodic table. The study of these neutron-rich superheavy nuclei is very interesting, because of their ground state structures and various mode of decays, including multi-fragment fission (more than two) [3]. Another interesting feature of some neutron rich uranium and thorium nuclei is that similar to ${ }^{233} \mathrm{U}$, ${ }^{235} \mathrm{U}$ and ${ }^{239} \mathrm{Pu}$, the nuclei ${ }^{246-264} \mathrm{U}$ and ${ }^{244-262} \mathrm{Th}$ are also thermally fissile, which are extremely important for the energy production in fission process. If the neutron rich uranium and

\footnotetext{
*Electronic address: bharat@iopb.res.in
}

thorium nuclei are the viable sources, then these nuclei will be more effective to achieve the critical condition in a controlled fission reaction.

Now the question arises, how we can get a reasonable estimation of the mass yield in the spallation reaction of these neutron rich thermally fissile nuclei. As mentioned earlier in this section, there are many formalisms available in the literature to study these cases. Here, we adopt the statistical model developed by Fong [5]. The calculation is further extended by Rajasekaran and Devanathan [6] to study the binary mass distributions using the single particle energies of the Nilsson model. The obtained results are well in agreement with the experimental data. In the present study, we would like to replace the single particle energies with the excitation energies of a successful microscopic approach, the relativistic mean field (RMF) formalism.

For last few decades, the relativistic mean field (RMF) formalism [7-11] with various parameter sets have successfully reproduced the bulk properties, such as binding energies, root mean square radii, quadrupole deformation etc. not only for nuclei near the $\beta$-stability line but also for nuclei away from it. Further, the RMF formalism is successfully applied to the study of clusterization of known cluster emitting heavy nucleus [12-14] and the fission of hyper-hyper deformed ${ }^{56} \mathrm{Ni}$ [15]. Rutz et. al. [16] reproduced the double, triple humped fission barrier of ${ }^{240} \mathrm{Pu},{ }^{232} \mathrm{Th}$ and the asymmetric ground states of ${ }^{226} \mathrm{Ra}$ using RMF formalism. Moreover, the symmetric and asymmetric fission modes are also successfully reproduced. Patra et. al. [3] studied the neck configuration in the fission decay of neutron rich $\mathrm{U}$ and $\mathrm{Th}$ isotopes. The main goal of this present paper is to understand the binary fragmentation yield of such neutron rich thermally fissile superheavy nuclei. ${ }^{250} \mathrm{U}$ and ${ }^{254} \mathrm{Th}$ are taken for further calculations as the representative cases.

The paper is organized as follows: In Section II, the statistical model and relativistic mean field theory are presented briefly. In subsection A of this section, the level density parameter and it's relation with the relative mass yield are outlined. In subsection B of II, the equation of motion of the nucleon and meson fields obtained from the relativistic mean field Lagrangian and the temperature dependent of the equa- 
tions are adopted through the occupation number of protons and neutrons. The results are discussed in Section III and compared with the finite range droplet model (FRDM) predictions. The summary and concluding remarks are given in Section IV.

\section{FORMALISM}

The possible binary fragments of the considered nucleus is obtained by equating the charge to mass ratio of the parent nucleus to the fission fragments as [17]:

$$
\frac{Z_{P}}{A_{P}} \approx \frac{Z_{i}}{A_{i}}
$$

with $A_{P}, Z_{P}$ and $A_{i}, Z_{i}(i=1$ and 2$)$ correspond to mass and charge numbers of the parent nucleus and the fission fragments [6]. The constraints, $A_{1}+A_{2}=A, Z_{1}+Z_{2}=Z$ and $A_{1} \geq A_{2}$ are imposed to satisfy the conservation of charge and mass number in a nuclear fission process and to avoid the repetition of fission fragments. Another constraint i.e., the binary charge numbers from $Z_{2} \geq 26$ to $Z_{1} \leq 66$ is also taken into consideration from the experimental yield [18] to generate the combinations, assuming that the fission fragments lie within these charge range.

\section{A. Statistical theory}

The statistical theory $[5,19]$ assumes that the probability of the particular fragmentation is directly proportional to the folded level density $\rho_{12}$ of that fragments with the total excitation energy $E^{*}$, i.e., $P\left(A_{j}, Z_{j}\right) \propto \rho_{12}\left(E^{*}\right)$. Where,

$$
\rho_{12}\left(E^{*}\right)=\int_{0}^{E^{*}} \rho_{1}\left(E_{1}^{*}\right) \rho_{2}\left(E^{*}-E_{1}^{*}\right) d E_{1}^{*},
$$

and $\rho_{i}$ is the level density of two fragments $(i=1,2)$. The nuclear level density $[20,21]$ is expressed as a function of fragment excitation energy $E_{i}^{*}$ and the single particle level density parameter $a_{i}$ which is given as:

$$
\rho_{i}\left(E_{i}^{*}\right)=\frac{1}{12}\left(\frac{\pi^{2}}{a_{i}}\right)^{1 / 4} E_{i}^{*(-5 / 4)} \exp \left(2 \sqrt{a_{i} E_{i}^{*}}\right) .
$$

In Refs. [17, 22], we calculate the excitation energies of the fragments using the ground state single particle energies of finite range droplet model (FRDM) [23] at a given temperature $T$ keeping the total number of proton and neutron fixed. In the present study, we apply the self consistent temperature dependent relativistic mean field theory to calculate the $E^{*}$ of the fragments. The excitation energy is calculated as,

$$
E_{i}^{*}(T)=E_{i}(T)-E_{i}(T=0) .
$$

The level density parameter $a_{i}$ is given as,

$$
a_{i}=\frac{E_{i}^{*}}{T^{2}} .
$$

The relative yield is calculated as the ratio of the probability of a given binary fragmentation to the sum of the probabilities of all the possible binary fragmentations and it is given by,

$$
Y\left(A_{j}, Z_{j}\right)=\frac{P\left(A_{j}, Z_{j}\right)}{\sum_{j} P\left(A_{j}, Z_{j}\right)},
$$

where $A_{j}$ and $Z_{j}$ are referred to the binary fragmentations involving two fragments with mass and charge numbers $A_{1}$, $A_{2}$ and $Z_{1}, Z_{2}$ obtained from Eq. (1). The competing basic decay modes such as neutron/proton emission, $\alpha$ decay and ternary fragmentation are not considered. In addition to these approximations, we have also not included the dynamics of the fission reaction, which are really important to get a quantitative comparison with the experimental measurements. The presented results are the prompt disintegration of a parent nucleus into two fragments (democratic breakup). The resulting excitation energy would be liberated as prompt particle emission or delayed emission, but such secondary emissions are also ignored.

\section{B. RMF Formalism}

The RMF theory assume that the nucleons interact with each other via meson fields. The nucleon - meson interaction is given by the Lagrangian density [7-9, 11, 24, 25],

$$
\begin{aligned}
\mathcal{L}= & \overline{\psi_{i}}\left\{i \gamma^{\mu} \partial_{\mu}-M\right\} \psi_{i}+\frac{1}{2} \partial^{\mu} \sigma \partial_{\mu} \sigma-\frac{1}{2} m_{\sigma}^{2} \sigma^{2} \\
& -\frac{1}{3} g_{2} \sigma^{3}-\frac{1}{4} g_{3} \sigma^{4}-g_{\sigma} \overline{\psi_{i}} \psi_{i} \sigma \\
& -\frac{1}{4} \Omega^{\mu \nu} \Omega_{\mu \nu}+\frac{1}{2} m_{w}^{2} V^{\mu} V_{\mu}-g_{w} \bar{\psi}_{i} \gamma^{\mu} \psi_{i} V_{\mu} \\
& -\frac{1}{4} \vec{B}^{\mu \nu} \cdot \vec{B}_{\mu \nu}+\frac{1}{2} m_{\rho}^{2} \vec{R}^{\mu} \cdot \vec{R}_{\mu}-g_{\rho} \bar{\psi}_{i} \gamma^{\mu} \vec{\tau} \psi_{i} \cdot \overrightarrow{R^{\mu}} \\
& -\frac{1}{4} F^{\mu \nu} F_{\mu \nu}-e \bar{\psi}_{i} \gamma^{\mu} \frac{\left(1-\tau_{3 i}\right)}{2} \psi_{i} A_{\mu} .
\end{aligned}
$$

Where, $\psi_{i}$ is the single particle Dirac spinor. The arrows over the letters in the above equation represent the isovector quantities. The nucleon, the $\sigma, \omega$, and $\rho$ meson masses are denoted by $\mathrm{M}, m_{\sigma}, m_{\omega}$ and $m_{\rho}$ respectively. The meson and the photon fields are termed as $\sigma, V_{\mu}, R^{\mu}$ and $A_{\mu}$ for $\sigma, \omega, \rho$ - mesons and photon respectively. The $g_{\sigma}, g_{\omega}, g_{\rho}$ and $\frac{e^{2}}{4 \pi}$ are the coupling constants for the $\sigma, \omega, \rho$-mesons and photon fields with nucleons respectively. The strength of the constants $g_{2}$ and $g_{3}$ are responsible for the nonlinear couplings of $\sigma$ meson $\left(\sigma^{3}\right.$ and $\left.\sigma^{4}\right)$. The field tensors of the isovector mesons and the photon are given by,

$$
\begin{aligned}
& \Omega^{\mu \nu}=\partial^{\mu} V^{\nu}-\partial^{\nu} V^{\mu} \\
& \vec{B}^{\mu \nu}=\partial^{\mu} \vec{R}^{\nu}-\partial^{\nu} \vec{R}^{\mu}-g_{\rho}\left(\vec{R}^{\mu} \times \vec{R}^{\nu}\right), \\
& F^{\mu \nu}=\partial^{\mu} A^{\nu}-\partial^{\nu} A^{\mu} .
\end{aligned}
$$

The classical variational principle gives the Euler-Lagrange equation and we get the Dirac-equation with potential terms 
for the nucleons and Klein-Gordan equations with source terms for the mesons. We assume the no-sea approximation, so we neglect the antiparticle states. We are dealing with the static nucleus, so the time reversal symmetry and the conservation of parity simplifies the calculations. After simplifications, the Dirac equation for the nucleon is given by,

$$
\{-i \alpha \cdot \nabla+V(r)+\beta[M+S(r)]\} \psi_{i}=\epsilon_{i} \psi_{i},
$$

where $\mathrm{V}(\mathrm{r})$ represents the vector potential and $\mathrm{S}(\mathrm{r})$ is the scalar potential,

$$
\begin{aligned}
V(r) & =g_{\omega} \omega_{0}+g_{\rho} \tau_{3} \rho_{0}(r)+e \frac{\left(1-\tau_{3}\right)}{2} A_{0}(r), \\
S(r) & =g_{\sigma} \sigma(r),
\end{aligned}
$$

which contributes to the effective mass,

$$
M^{*}(r)=M+S(r) .
$$

The Klein-Gordon equations for the mesons and the electromagnetic fields with the nucleon densities as sources are,

$$
\begin{gathered}
\left\{-\triangle+m_{\sigma}^{2}\right\} \sigma(r)=-g_{\sigma} \rho_{s}(r)-g_{2} \sigma^{2}(r)-g_{3} \sigma^{3}(r), \\
\left\{-\triangle+m_{\omega}^{2}\right\} \omega_{0}(r)=g_{\omega} \rho_{v}(r) \\
\left\{-\triangle+m_{\rho}^{2}\right\} \rho_{0}(r)=g_{\rho} \rho_{3}(r) \\
-\triangle A_{0}(r)=e \rho_{c}(r)
\end{gathered}
$$

The corresponding densities such as scalar, baryon (vector), isovector and proton (charge) are given as

$$
\begin{aligned}
\rho_{s}(r) & =\sum_{i} n_{i} \psi_{i}^{\dagger}(r) \psi_{i}(r), \\
\rho_{v}(r) & =\sum_{i} n_{i} \psi_{i}^{\dagger}(r) \gamma_{0} \psi_{i}(r), \\
\rho_{3}(r) & =\sum_{i} n_{i} \psi_{i}^{\dagger}(r) \tau_{3} \psi_{i}(r), \\
\rho_{\mathrm{c}}(r) & =\sum_{i} n_{i} \psi_{i}^{\dagger}(r)\left(\frac{1-\tau_{3}}{2}\right) \psi_{i}(r) .
\end{aligned}
$$

To solve the Dirac and Klein-Gordan equations, we expand the Boson fields and the Dirac spinor in an axially deformed harmonic oscillator basis with $\beta_{0}$ as the initial deformation parameter. The nucleon equation along with different meson equations form a set of coupled equations, which can be solved by iterative method. The center of mass correction is calculated with the non-relativistic approximation. The quadrupole deformation parameter $\beta_{2}$ is calculated from the resulting quadrupole moments of the proton and neutron. The total energy is given by $[10,26,27]$,

$$
\begin{gathered}
E(T)=\sum_{i} \epsilon_{i} n_{i}+E_{\sigma}+E_{\sigma N L}+E_{\omega}+E_{\rho} \\
+E_{C}+E_{\text {pair }}+E_{c . m .}-A M
\end{gathered}
$$

with

$$
\begin{gathered}
E_{\sigma}=-\frac{1}{2} g_{\sigma} \int d^{3} r \rho_{s}(r) \sigma(r), \\
E_{\sigma N L}=-\frac{1}{2} \int d^{3} r\left\{\frac{2}{3} g_{2} \sigma^{3}(r)+\frac{1}{2} g_{3} \sigma^{4}(r)\right\}, \\
E_{\omega}=-\frac{1}{2} g_{\omega} \int d^{3} r \rho_{v}(r) \omega^{0}(r), \\
E_{\rho}=-\frac{1}{2} g_{\rho} \int d^{3} r \rho_{3}(r) \rho^{0}(r), \\
E_{C}=-\frac{e^{2}}{8 \pi} \int d^{3} r \rho_{c}(r) A^{0}(r), \\
E_{\text {pair }}=-\triangle \sum_{i>0} u_{i} v_{i}=-\frac{\triangle^{2}}{G}, \\
E_{c . m .}=-\frac{3}{4} \times 41 A^{-1 / 3} .
\end{gathered}
$$

Here, $\epsilon_{i}$ is the single particle energy, $n_{i}$ is the occupation probability and $E_{\text {pair }}$ is the pairing energy obtained from the simple BCS formalism.

\section{Pairing and temperature dependent RMF formalism}

The pairing correlation plays a distinct role in open-shell nuclei. The effect of pairing correlation is markedly seen with increase in mass number A. Moreover it helps in understanding the deformation of medium and heavy nuclei. It has a lean effect on both bulk and single particles properties of lighter mass nuclei because of the availability of limited pairs near the Fermi surface. We take the case of $\mathrm{T}=1$ channel of pairing correlation i.e, pairing between proton- proton and neutronneutron. In this case, a nucleon of quantum states $\left|j m_{z}\right\rangle$ pairs with another nucleons having same $I_{z}$ value with quantum states $\left|j-m_{z}\right\rangle$, since it is the time reversal partner of the other. In both nuclear and atomic domain the ideology of BCS pairing is the same. The even-odd mass staggering of isotopes was the first evidence of its kind for the pairing energy. Considering the mean-field formalism, the violation of the particle number is seen only due to the pairing correlation. We find terms like $\psi^{\dagger} \psi$ (density) in the RMF Lagrangian density but we put an embargo on terms of the form $\psi^{\dagger} \psi^{\dagger}$ or $\psi \psi$ since it violates the particle number conservation. We apply externally the BCS constant pairing gap approximation for our calculation to take the pairing correlation into account. The pairing interaction energy in terms of occupation probabilities $v_{i}^{2}$ and $u_{i}^{2}=1-v_{i}^{2}$ is written as [28, 29]:

$$
E_{\text {pair }}=-G\left[\sum_{i>0} u_{i} v_{i}\right]^{2},
$$

with $G$ is the pairing force constant. The variational approach with respect to the occupation number $v_{i}^{2}$ gives the BCS equation [29]:

$$
2 \epsilon_{i} u_{i} v_{i}-\triangle\left(u_{i}^{2}-v_{i}^{2}\right)=0,
$$


with the pairing gap $\triangle=G \sum_{i>0} u_{i} v_{i}$. The pairing gap $(\triangle)$ of proton and neutron is taken from the empirical formula [10, 30]:

$$
\triangle=12 \times A^{-1 / 2} .
$$

The temperature introduced in the partial occupancies in the $\mathrm{BCS}$ approximation is given by,

$$
n_{i}=v_{i}^{2}=\frac{1}{2}\left[1-\frac{\epsilon_{i}-\lambda}{\tilde{\epsilon}_{i}}\left[1-2 f\left(\tilde{\epsilon}_{i}, T\right)\right]\right],
$$

with

$$
\begin{gathered}
f\left(\tilde{\epsilon}_{i}, T\right)=\frac{1}{\left(1+\exp \left[\tilde{\epsilon}_{i} / T\right]\right)} \text { and } \\
\tilde{\epsilon}_{i}=\sqrt{\left(\epsilon_{i}-\lambda\right)^{2}+\triangle^{2}} .
\end{gathered}
$$

The function $f\left(\tilde{\epsilon}_{i}, T\right)$ represents the Fermi Dirac distribution for quasi particle energy $\tilde{\epsilon}_{i}$. The chemical potential $\lambda_{p}\left(\lambda_{n}\right)$ for protons (neutrons) is obtained from the constraints of particle number equations

$$
\begin{aligned}
& \sum_{i} n_{i}^{Z}=Z \\
& \sum_{i} n_{i}^{N}=N .
\end{aligned}
$$

The sum is taken over all proton and neutron states. The entropy is obtained by,

$$
S=-\sum_{i}\left[n_{i} \ln \left(n_{i}\right)+\left(1-n_{i}\right) \ln \left(1-n_{i}\right)\right] .
$$

The total energy and the gap parameter are obtained by minimizing the free energy,

$$
F=E-T S
$$

In constant pairing gap calculations, for a particular value of pairing gap $\triangle$ and force constant $G$, the pairing energy $E_{\text {pair }}$ diverges, if it is extended to an infinite configuration space. In fact, in all realistic calculations with finite range forces, $\triangle$ is not constant, but decreases with large angular momenta states above the Fermi surface. Therefore, a pairing window in all the equations are extended up-to the level $\left|\epsilon_{i}-\lambda\right| \leq$ $2\left(41 A^{-1 / 3}\right)$ as a function of the single particle energy. The factor 2 has been determined so as to reproduce the pairing correlation energy for neutrons in ${ }^{118} \mathrm{Sn}$ using Gogny force $[10,28,31]$.

\section{RESULTS AND DISCUSSIONS}

In our very recent work [32], we have calculated the ternary mass distributions for ${ }^{252} \mathrm{Cf},{ }^{242} \mathrm{Pu}$ and ${ }^{236} \mathrm{U}$ with the fixed third fragments $\mathrm{A}_{3}={ }^{48} \mathrm{Ca},{ }^{20} \mathrm{O}$ and ${ }^{16} \mathrm{O}$ respectively for the three different temperatures $\mathrm{T}=1,2$ and $3 \mathrm{MeV}$ within the TRMF formalism. The structure effects of binary fragments are also reported in Ref. [33]. In this article, we study the mass distribution of ${ }^{250} \mathrm{U}$ and ${ }^{254} \mathrm{Th}$ as a representative cases from the range of neutron-rich thermally fissile nuclei ${ }^{246-264} \mathrm{U}$ and ${ }^{244-262} \mathrm{Th}$. Because of the neutron-rich nature of these nuclei, a large number of neutrons emit during the fission process. These nucleons help to achieve the critical condition much sooner than the normal fissile nuclei.

To assure the predictability of the statistical model, we also study the binary fragmentation of naturally occurring ${ }^{236} \mathrm{U}$ and ${ }^{232}$ Th nuclei. The possible binary fragments are obtained using the Eq. (1). To calculate the total binding energy at a given temperature, we use the axially symmetric harmonic oscillator basis expansion $\mathrm{N}_{F}$ and $\mathrm{N}_{B}$ for the Fermion and Boson wave-functions to solve the Dirac Eq. (11) and the Klein Gordon Eqs. (14 - 17) iteratively. It is reported [34] that the effect of basis space on the calculated binding energy, quadrupole deformation parameter $\left(\beta_{2}\right)$ and the rms radii of nucleus are almost equal for the basis set $\mathrm{N}_{F}=\mathrm{N}_{B}=12$ to 20 in the mass region $\mathrm{A} \sim 200$. Thus, we use the basis space $\mathrm{N}_{F}=12$ and $\mathrm{N}_{B}=20$ to study the binary fragments up to mass number $A \sim 182$. The binding energy is obtained by minimizing the free energy, which gives the most probable quadrupole deformation parameter $\beta_{2}$ and the proton (neutron) pairing gaps $\triangle_{p}\left(\triangle_{n}\right)$ for the given temperature. At finite temperature, the continuum corrections due to the excitation of nucleons to be considered. The level density in the continuum depends on the basis space $\mathrm{N}_{F}$ and $\mathrm{N}_{B}$ [35]. It is shown that the continuum corrections need not be included in the calculations of level densities up-to the temperature $\mathrm{T} \sim 3$ $\mathrm{MeV}[36,37]$.

\section{A. Level density parameter and level density within TRMF and FRDM formalisms}

In TRMF, the excitation energies $E^{*}$ and the level density parameters $a_{i}$ of the fragments are obtained self consistently from Eqns. (4) to (5). The FRDM calculations are also done for comparison. In this case, level density of the fragments are evaluated from the ground state single particle energies of the finite range droplet model (FRDM) of Möller et. al. [38] which are retrieved from the Reference Input Parameter Library (RIPL-3) [39]. The total energy at a given temperature is calculated as $E(T)=\sum n_{i} \epsilon_{i} ; \epsilon_{i}$ are the ground state single particle energies and $n_{i}$ are the Fermi-Dirac distribution function. The $T$ dependent energies are obtained by varying the occupation numbers at a fixed particle number for a given temperature and given fragment. The level density parameter $a$ is a crucial quantity in the statistical theory for the estimation of yields. These values of $a$ for the binary fragments of ${ }^{236} \mathrm{U},{ }^{250} \mathrm{U},{ }^{232} \mathrm{Th}$ and ${ }^{254} \mathrm{Th}$ obtained from TRMF and FRDM are depicted in Fig. 1. The empirical estimation $a=A / K$ are also given for comparison, with $K$, the inverse level density parameter. In general, the $K$ value varies from 8 to 13 with the increasing temperature. However, the level density parameter is considered to be constant up-to $T \approx 4 \mathrm{MeV}$. Hence, we take the practical value of $K=10$ as mentioned 


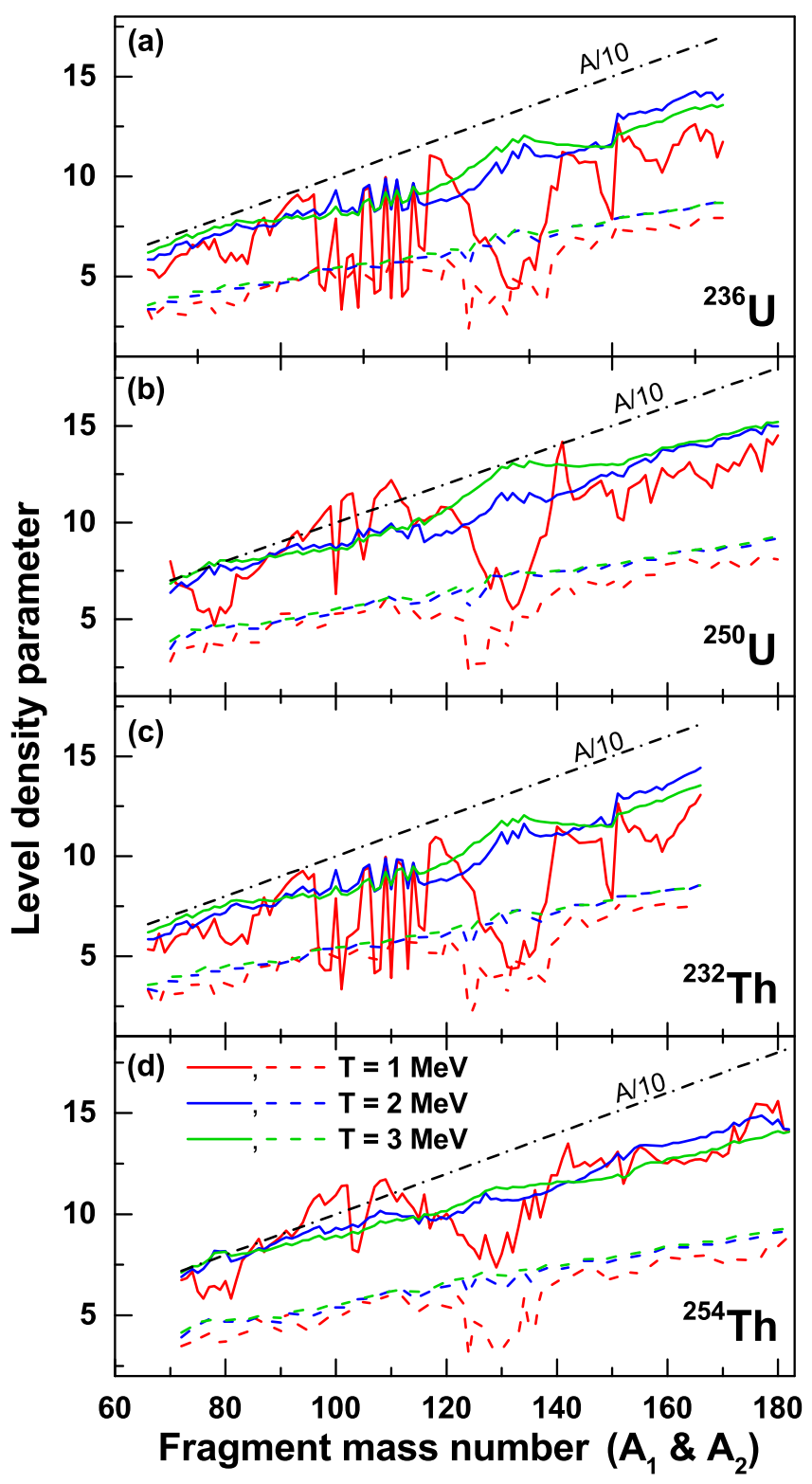

FIG. 1: (Color online) The level density parameter $a$ for the binary fragmentation of ${ }^{236} \mathrm{U},{ }^{250} \mathrm{U},{ }^{232} \mathrm{Th}$ and ${ }^{254} \mathrm{Th}$ at temperature $T=1$, 2 and $3 \mathrm{MeV}$ within the TRMF (solid lines) and FRDM (dash lines) formalisms.

in Ref. [40]. The $a$ values of TRMF are close to the empirical level density parameter. The FRDM level density parameters are appreciably lower than the referenced $a$. Further, in both models at $T=1 \mathrm{MeV}$, there are more fluctuations in the level density parameter due to the shell effects of the fragments. At $T=2$ and $3 \mathrm{MeV}$, the variations are small. This may be due to the fact that the shell become degenerate at the higher temperatures. All fragments becomes spherical at temperature $T \approx$ $3 \mathrm{MeV}$ as shown in Ref. [33]. The level density parameter $a$ is evaluated in two different ways using excitation energy and

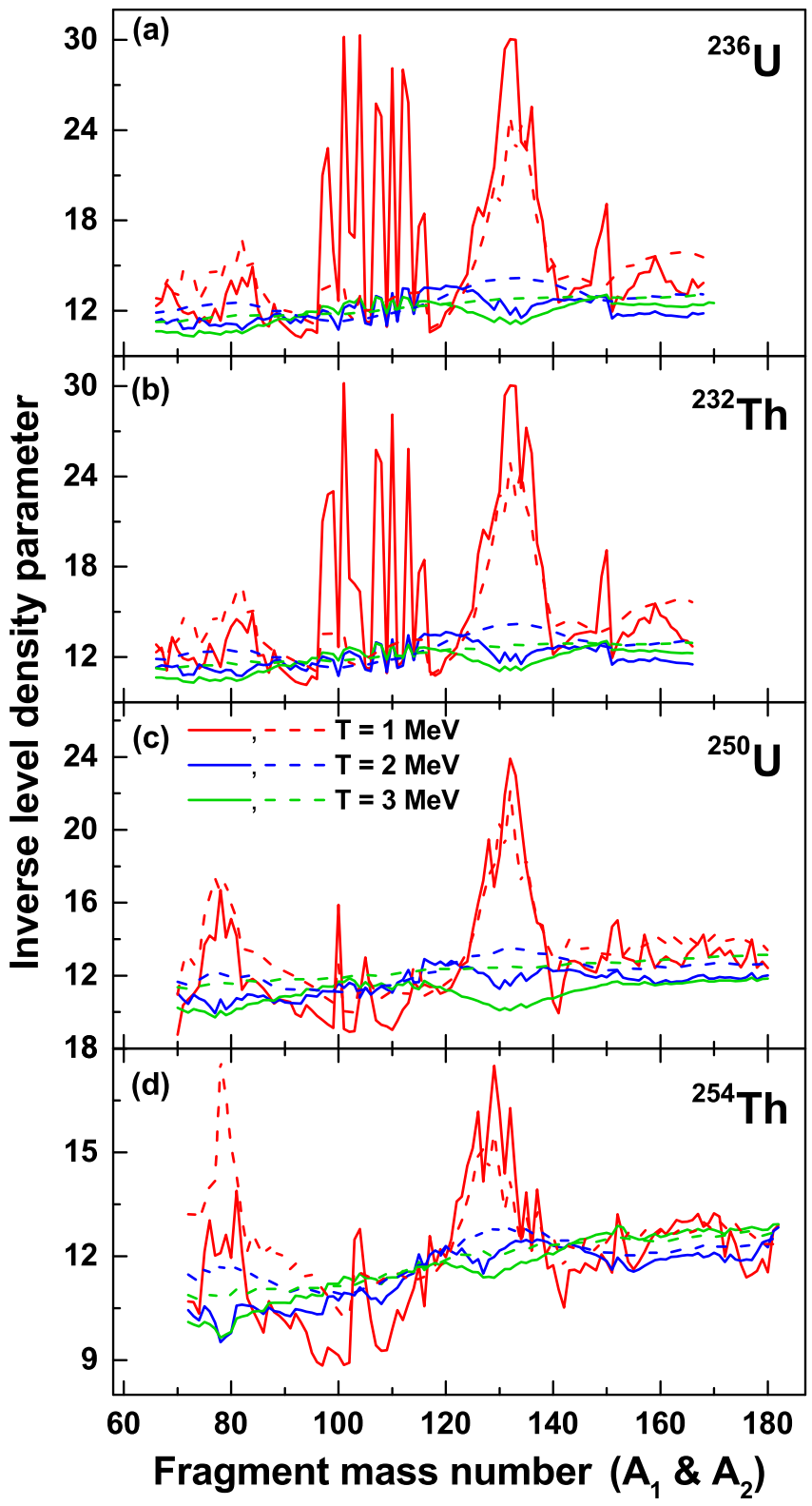

FIG. 2: (Color online) The inverse level density parameters $K_{E}$ (solid lines) and $K_{S}$ (dash lines) are obtained for ${ }^{236} \mathrm{U},{ }^{250} \mathrm{U},{ }^{232} \mathrm{Th}$ and ${ }^{254} \mathrm{Th}$ at temperatures $T=1,2$ and $3 \mathrm{MeV}$.

the entropy of the system as:

$$
\begin{aligned}
a_{E} & =\frac{E^{*}}{T^{2}}, \\
a_{S} & =\frac{S}{2 T} .
\end{aligned}
$$

For instance, the inverse level density parameters $K_{E}$ and $K_{S}$ of ${ }^{236} \mathrm{U},{ }^{250} \mathrm{U},{ }^{232} \mathrm{Th}$ and ${ }^{254} \mathrm{Th}$ within TRMF formalism are depicted in Fig. 2. Both $K_{S}$ and $K_{E}$ have maximum fluctuation upto $30 \mathrm{MeV}$ at $T=1 \mathrm{MeV}$. These values reduce to $10-13 \mathrm{MeV}$ at temperature $T=2 \mathrm{MeV}$ or above. It is to be noted that at $T=3 \mathrm{MeV}$, the inverse level density parameter substantially lower around the mass number $A \sim 130$ in all 


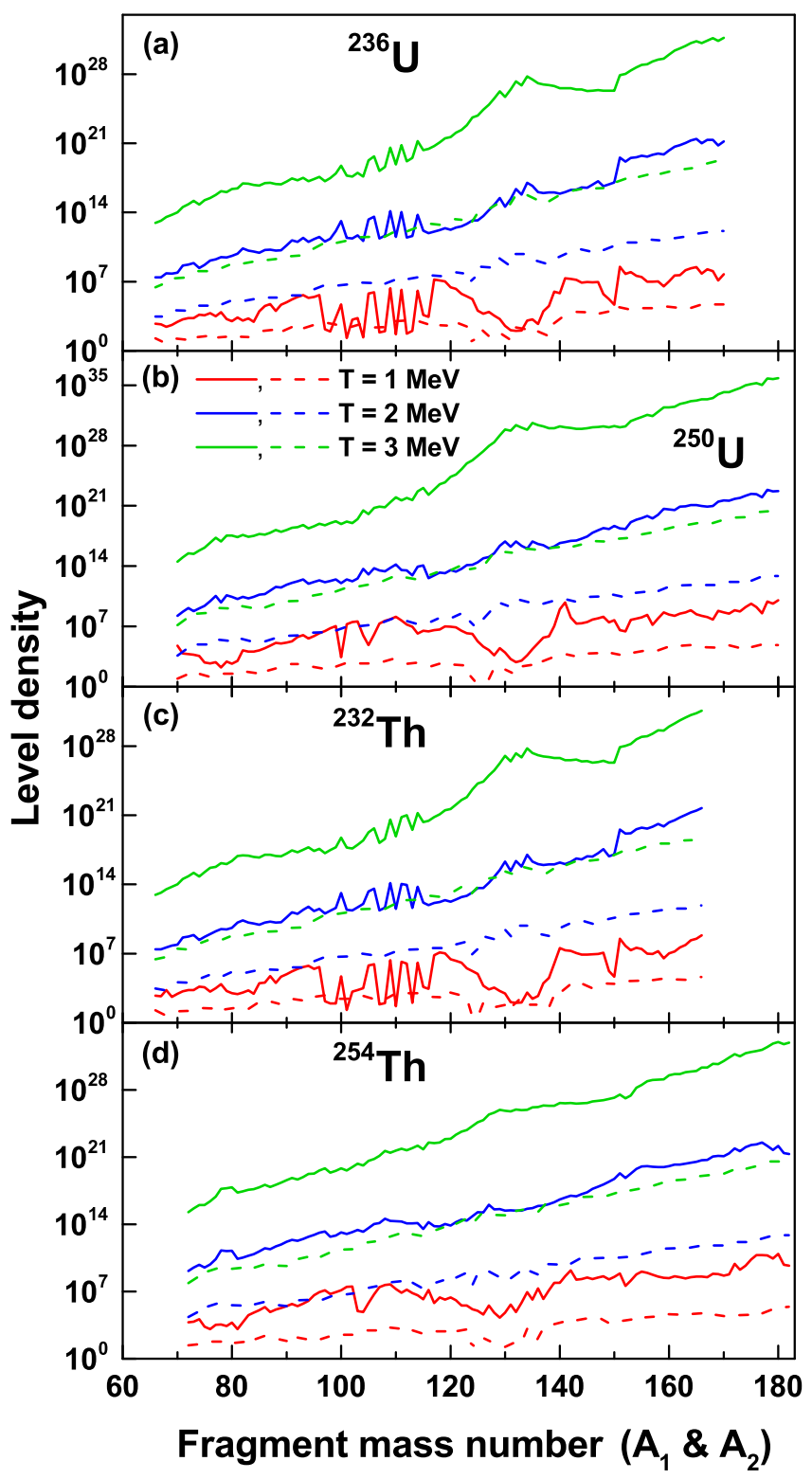

FIG. 3: (Color online) The level density of the binary fragmentations of ${ }^{236} \mathrm{U},{ }^{250} \mathrm{U},{ }^{232} \mathrm{Th}$ and ${ }^{254} \mathrm{Th}$ at temperature $T=1,2$ and $3 \mathrm{MeV}$ within the TRMF (solid lines) and FRDM (dash lines) formalisms.

cases. This may be due to the neutron closed shell $(N=82)$ in the fission fragments of ${ }^{236} \mathrm{U}$ and ${ }^{232} \mathrm{Th}$ and the neutron-rich nuclei ${ }^{250} \mathrm{U}$ and ${ }^{254} \mathrm{Th}$. The level density for the fission fragments of ${ }^{236} \mathrm{U},{ }^{250} \mathrm{U},{ }^{232} \mathrm{Th}$ and ${ }^{254} \mathrm{Th}$ are plotted as a function of mass number in Fig. 3 within the TRMF and FRDM formalisms at three different temperatures $T=1,2$ and $3 \mathrm{MeV}$.

The level density $\rho$ has maximum fluctuations at $T=1$ $\mathrm{MeV}$ for all considered nuclei in TRMF model similar to the level density parameter $a$. The $\rho$ values are substantially lower at mass number $A \sim 130$ for all nuclei. In Fig. 3, one can notice that the level density has small kinks in the mass region $A \sim 71-81$ of ${ }^{236} \mathrm{U}$ and $A \sim 77-91$ of ${ }^{250} \mathrm{U}$, comparing with the neighboring nuclei at temperature $T=2 \mathrm{MeV}$. Consequently, the corresponding partner fragments have also higher $\rho$ values. A further inspection reveals that the level density of the closed shell nucleus around $A \sim 130$ has higher value than the neighboring nuclei for both ${ }^{236,250} \mathrm{U}$, but it has lower yield due to the smaller level density of the corresponding partners. At $T=3 \mathrm{MeV}$, the level density of the fragments around mass number $A \sim 72$ and 130 have larger values compared to other fragments of ${ }^{236} \mathrm{U}$. On the other hand, the level density in the vicinity of neutron number $N=82$ and proton number $Z=50$ for the fragments of the neutron-rich ${ }^{250} \mathrm{U}$ nucleus is quite high, because of the close shell of the fragments. This is evident from the small kink in the level density of ${ }^{130} \mathrm{Cd}(N=82),{ }^{132} \mathrm{In}(N \sim 82)$ and ${ }^{135} \mathrm{Sn}(Z=50)$. Again, for ${ }^{232} \mathrm{Th}$, the level densities are found to be maximum at around mass number $A \sim 81$ and 100 for $T=2 \mathrm{MeV}$. In case of ${ }^{254} \mathrm{Th}$, the $\rho$ values are found to be large for the fragments around $A \sim 78$ and 97 at $T=2 \mathrm{MeV}$. Their corresponding partners have also similar behavior. For higher temperature $T=3 \mathrm{MeV}$, the higher $\rho$ values of ${ }^{232} \mathrm{Th}$ fragments are notable around mass number $A \sim 130$. Similarly, for ${ }^{254} \mathrm{Th}$, the fission fragments around $A \sim 78$ has higher level density at $T=3 \mathrm{MeV}$. In general, the level density increases towards the neutron closed shell $(N=82)$ nucleus.

\section{B. Relative fragmentation distribution in binary systems}

In this section, the mass distributions of ${ }^{236} \mathrm{U},{ }^{232} \mathrm{Th}$ and the neutron rich nuclei ${ }^{250} \mathrm{U}$ and ${ }^{254} \mathrm{Th}$ are calculated at temperatures $T=1,2$ and $3 \mathrm{MeV}$ using TRMF and FRDM excitation energies and the level density parameters $a$ as explained in Sec. II. The binary mass distributions of ${ }^{236,250} \mathrm{U}$ and ${ }^{232,254}$ Th are plotted in Figs. 4 and 5. The total energy at finite temperature and ground state energy are calculated using the TRMF formalism as discussed in the section III A. From the excitation energy $\mathrm{E}^{*}$ and the temperature $T$, the level density parameter $a$ and the level density $\rho$ of the fragments are calculated using Eq. 3. From the fragment level densities $\rho_{i}$, the folding density $\rho_{12}$ is calculated using the convolution integral as in Eq. 2 and the relative yields are calculated using Eq. 6 . The total yields are normalized to the scale 2 .

The mass yield of normal nuclei ${ }^{236} \mathrm{U}$ and ${ }^{232} \mathrm{Th}$ are briefly explains first, followed by the detailed description of the neutron rich nuclei. The results of most favorable fragments yield of ${ }^{236,250} \mathrm{U}$ and ${ }^{232,254} \mathrm{Th}$ are listed in Table I at three different temperatures $T=1,2$ and $3 \mathrm{MeV}$ for both TRMF and FRDM formalisms. From Figs. 4 and 5, it is shown that the mass distributions for ${ }^{236} \mathrm{U}$ and ${ }^{232} \mathrm{Th}$ are quite different than the neutron-rich ${ }^{250} \mathrm{U}$ and ${ }^{254} \mathrm{Th}$ isotopes.

The symmetric binary fragmentation ${ }^{118} \mathrm{Pd}+{ }^{118} \mathrm{Pd}$ for ${ }^{236} \mathrm{U}$ is the most favorable combination. In TRMF, the fragments with close shell $(N=100$ and $Z=28)$ combinations are more probable at the temperature $T=2 \mathrm{MeV}$. The blend region of neutron and proton close shell $(N \approx 82$ and $Z \approx 50)$ has the considerable yield values at $T=3 \mathrm{MeV}$. The fragmentations ${ }^{151} \mathrm{Pr}+{ }^{85} \mathrm{As},{ }^{142} \mathrm{Cs}+{ }^{94} \mathrm{Rb}$ and ${ }^{144} \mathrm{Ba}+{ }^{92} \mathrm{Kr}$ are the favorable combinations at temperature $T=1 \mathrm{MeV}$ in FRDM formalism. For higher temperatures $T=2$ and $3 \mathrm{MeV}$, the closed shell or near closed shell fragments $(N=82,50$ and 


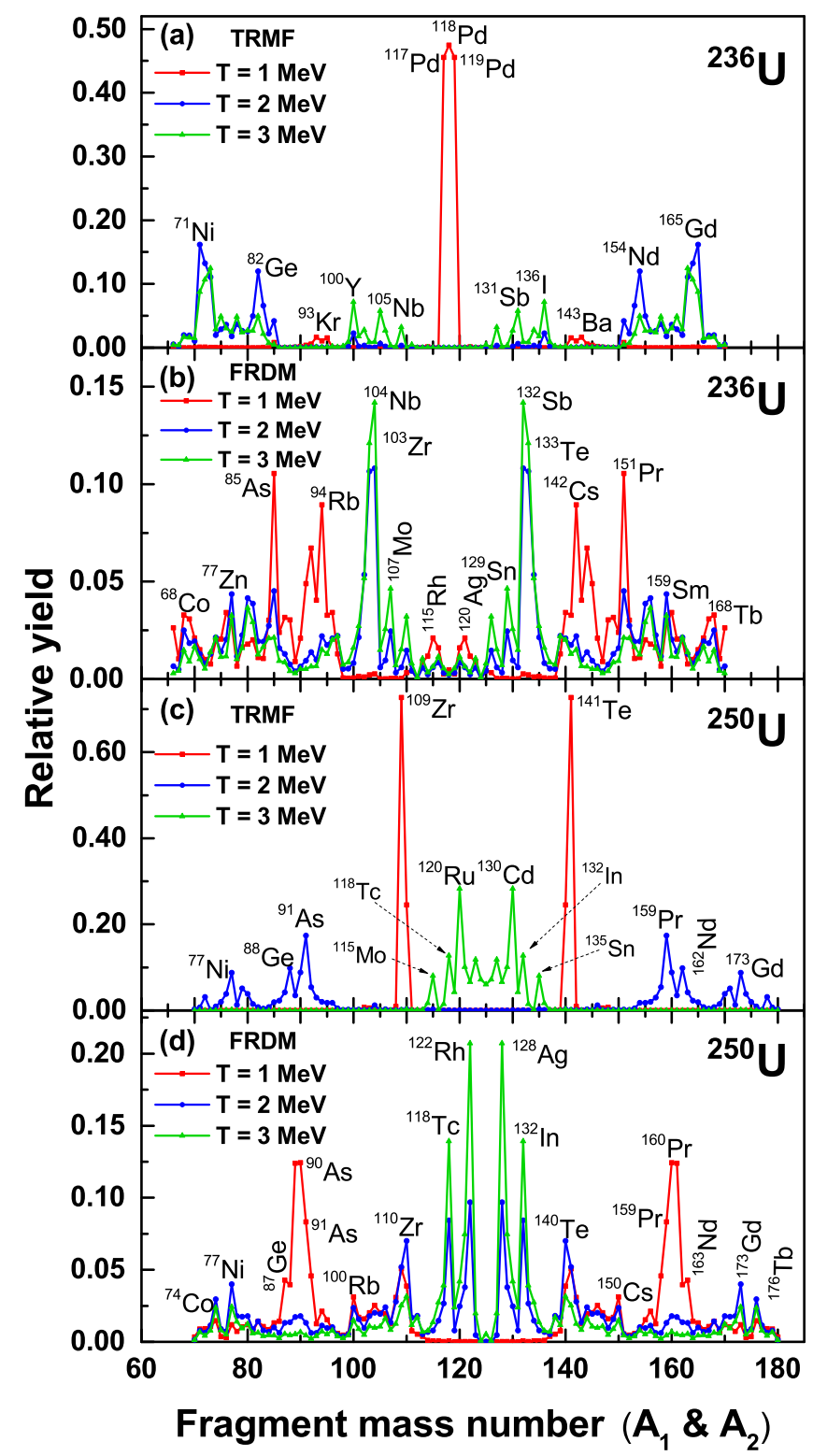

FIG. 4: (Color online) Mass distribution of ${ }^{236} U$ and ${ }^{250} U$ at temperatures $\mathrm{T}=1,2$ and $3 \mathrm{MeV}$. The total yield values are normalized to the scale 2 .

$Z=28$ ) have larger yields. From Fig. 5 in TRMF formalism, the combinations ${ }^{118} \mathrm{Pd}+{ }^{114} \mathrm{Ru}$ and ${ }^{140} \mathrm{Xe}+{ }^{92} \mathrm{Kr}$ are the possible fragments at $T=1 \mathrm{MeV}$ for the nucleus ${ }^{232} \mathrm{Th}$. At $T=$ $2 \mathrm{MeV}$, we find maximum yields for the fragments with the close shell or near close shell combinations $(N=82,50)$. For higher temperature $T=3 \mathrm{MeV}$, near the neutron close shell $(N \sim 82),{ }^{132} \mathrm{Sb}+{ }^{100} \mathrm{Y}$ is the most favorable fragmentation pair compared with all other yields. Similar fragmentations are found in the FRDM formalism at $T=2$ and $3 \mathrm{MeV}$. In addition, the probability of the evaluation of ${ }^{129} \mathrm{Sn}+{ }^{103} \mathrm{Zr}$ is also quite substantial in the fission process. For $T=1 \mathrm{MeV}$, the yield is more or less similar with the TRMF model.

From Fig. 4, for ${ }^{250} \mathrm{U}$ the fragment combinations ${ }^{140,141} \mathrm{Te}$

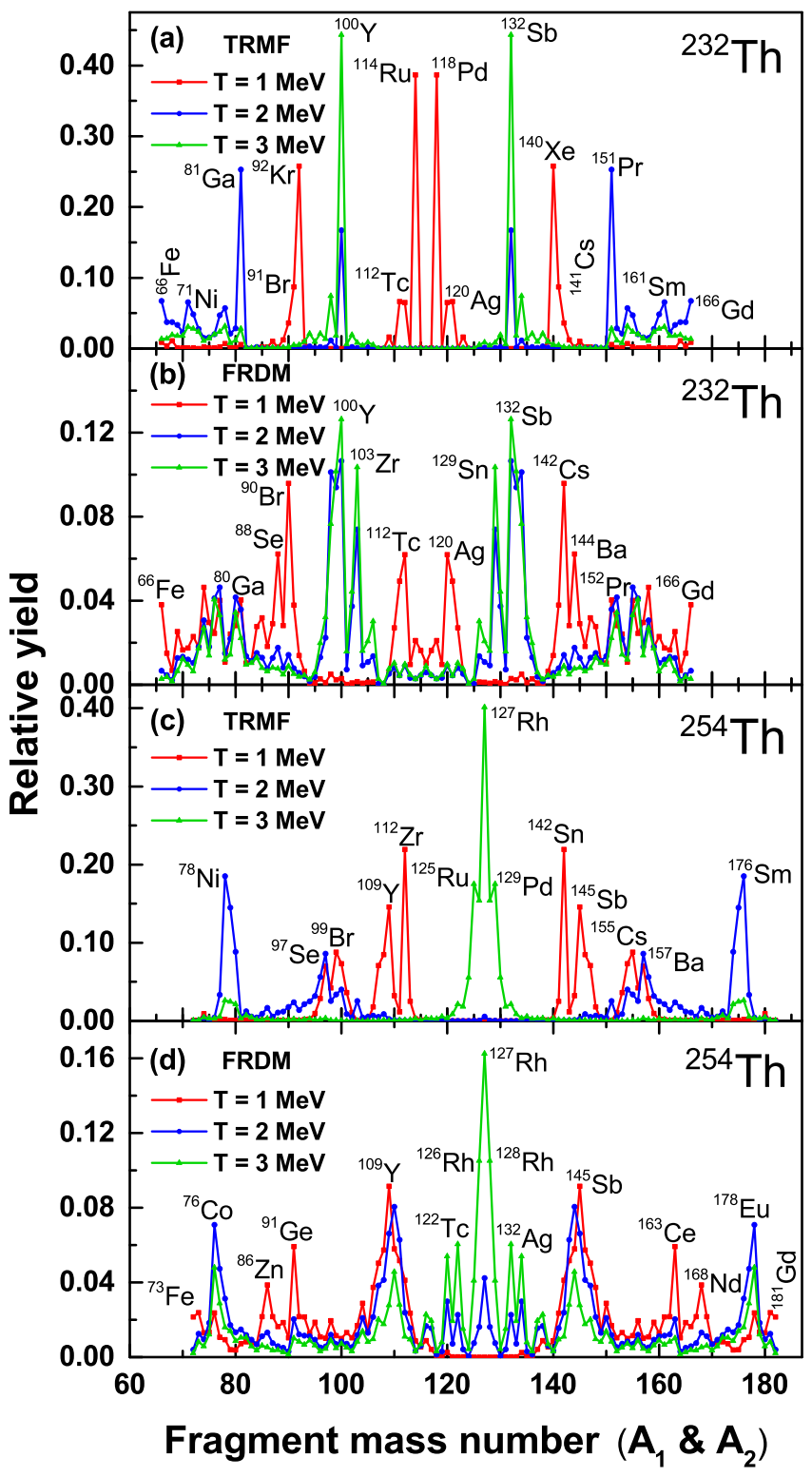

FIG. 5: (Color online) Mass distribution of ${ }^{232} \mathrm{Th}$ and ${ }^{254} \mathrm{Th}$ at temperatures $\mathrm{T}=1,2$ and $3 \mathrm{MeV}$. The total yield values are normalized to the scale 2 .

$+{ }^{110,109} \mathrm{Zr}$ have the maximum yields at $T=1 \mathrm{MeV}$ in TRMF. This is also consistent with the evolution of the sub-close shell proton $Z=40$ in $Z r$ isotopes [41]. Contrary to this almost symmetric binary yield, the mass distribution of this nucleus in FRDM formalism have the asymmetric evolution of the fragment combinations like ${ }^{160,159} \mathrm{Pr}+{ }^{90,91} \mathrm{As},{ }^{163,162} \mathrm{Nd}$ $+{ }^{87,88} \mathrm{Ge}$ and ${ }^{150} \mathrm{Cs}+{ }^{100} \mathrm{Rb}$. Interestingly, at $T=2$ and $3 \mathrm{MeV}$, the more favorable fragment combinations have one of the closed shell nuclei. At $T=2 \mathrm{MeV},{ }^{159} \mathrm{Pr}+{ }^{91} \mathrm{As}$, ${ }^{162} \mathrm{Nd}+{ }^{88} \mathrm{Ge}$ and ${ }^{173} \mathrm{Gd}+{ }^{77} \mathrm{Ni}$ are the more probable fragmentations (see Fig. 4(c)). It is reported by Satpathy et al [42] and experimentally verified by Patel et al [43] that $N=100$ is a neutron close shell for the deformed region, where $Z=62$ acts like a magic number. In FRDM, ${ }^{128} \mathrm{Ag}$ 
$+{ }^{122} \mathrm{Rh},{ }^{132} \mathrm{In}+{ }^{118} \mathrm{Tc},{ }^{140} \mathrm{Te}+{ }^{110} \mathrm{Zr}$ and ${ }^{173} \mathrm{Gd}+{ }^{77} \mathrm{Ni}$ have larger yield at temperature $T=2 \mathrm{MeV}$. In TRMF method, the most favorable fragments are confined in the single region $(A \approx 114-136)$ which is the blend of vicinity of neutron $(N=82)$ and proton $(Z=50)$ closed shell nuclei at $T=3 \mathrm{MeV}$. The fragment combinations ${ }^{130} \mathrm{Cd}+{ }^{120} \mathrm{Ru},{ }^{132} \mathrm{In}$ $+{ }^{118} \mathrm{Tc}$ and ${ }^{135} \mathrm{Sn}+{ }^{115} \mathrm{Mo}$ are the major yields for ${ }^{250} \mathrm{U}$ at $\mathrm{T}=3 \mathrm{MeV}$ in TRMF calculations. In FRDM method, at $T=3$ $\mathrm{MeV}$, more probable fragments are similar that at $T=2 \mathrm{MeV}$. A comparison between Fig. 4(c) and 4(d) clears that, although the prediction of FRDM and TRMF at $T=3 \mathrm{MeV}$ are qualitatively similar, but it is quantitatively very different at $T=2$ $\mathrm{MeV}$ in both the predictions. Also, from Fig. 4, it is inferred that the yields of the fragment combinations in blend region increases and in other regions decreases at $T=2 \mathrm{MeV}$.

In the present study, the total energy of the parent nucleus A is more than the sum of the energies of the daughters $A_{1}$ and $A_{2}$. Here, the dynamics of entire process starting from the initial stage upto the scission are ignored. As a result, the energy conservation in the spallation reaction does not taken into account. The fragment yield can be regarded as the relative fragmentation probability, which is obtained from Eq. 6. Now we analyze the fragmentation yields for Th isotopes and the results are depicted in Fig. 5 and Table I. In this case, one can see that the mass distribution broadly spreads through out the region $A_{i}=66-166$. Again, the most concentrated yields can be divided into two regions $\mathrm{I}\left(A_{1}=141-148\right.$ and $\left.A_{2}=106-113\right)$ and II $\left(A_{1}=152-158\right.$ and $A_{2}=102-$ 96) for ${ }^{254} \mathrm{Th}$ in TRMF formalism at the temperature $T=1$ $\mathrm{MeV}$. The most favorable fragmentation ${ }^{142} \mathrm{Sn}+{ }^{112} \mathrm{Zr}$ is obtained from region $\mathrm{I}$. The other combinations in that region have also considerable yields. In region II, the isotopes of $\mathrm{Ba}$ and $\mathrm{Cs}$ appears curiously along with their corresponding partners. Categorically, in FRDM predictions, region I has larger yields at $T=1 \mathrm{MeV}$. The other possible fragmentations are ${ }^{163} \mathrm{Ce}+{ }^{91} \mathrm{Ge},{ }^{168} \mathrm{Nd}+{ }^{86} \mathrm{Zn}$ and ${ }^{181} \mathrm{Gd}+{ }^{73} \mathrm{Fe}$ (See Fig. $5(b, d))$. The mass distribution is different with different temperature and the maximum yields at $T=2 \mathrm{MeV}$ in TRMF formalism are ${ }^{174,175,176} \mathrm{Sm}+{ }^{80,79,78} \mathrm{Ni}$. Apart from these combinations, there are other considerable yields can be seen in Fig. 5 for region II. The prediction of maximum probability of the fragments production in FRDM method are ${ }^{144} \mathrm{Sb}+{ }^{110} \mathrm{Y},{ }^{178} \mathrm{Eu}+{ }^{76} \mathrm{Co}$ and ${ }^{127} \mathrm{Rh}+{ }^{127} \mathrm{Rh}$ at $T=2$ $\mathrm{MeV}$. Besides these yields, one can find other notable evolution of masses in region I due to the vicinity of the proton close shell. Interestingly, at $T=3 \mathrm{MeV}$, symmetric binary combination ${ }^{127} \mathrm{Rh}+{ }^{127} \mathrm{Rh}$ has the largest yield due to the neutron close shell $(N=82)$ of the fragment ${ }^{127} \mathrm{Rh}$. The other yield fragments have exactly/nearly a magic nucleon combination, mostly neutron $(N=82)$ as one of the fragment. A considerable yield is also seen for the proton close shell $(Z=$ 28) $\mathrm{Ni}$ or/and $(Z=62) \mathrm{Sm}$ isotopes supporting our earlier prediction [33]. This confirms the prediction of $\mathrm{Sm}$ as a deformed magic nucleus [42, 43]. Another observation of the present calculations show that the yields of the neutron-rich nuclei agree with the symmetric mass distribution of Chaudhuri et. al. [44] at large excitation energy, which contradict the recent prediction of large asymmetric mass distribution of neutron-deficient Th isotopes [45]. These two results [44, 45] along with our present calculations confirm that the symmetric or asymmetric mass distribution at different temperature depends on the proton and neutron combination of the parent nucleus. In general, both TRMF and FRDM predict maximum yields for both symmetric/asymmetric binary fragmentations followed by other secondary fragmentations emission depending on the temperature as well as the mass number of the parent nucleus. Thus, the binary fragments have larger level density $\rho$ comparing with other nuclei because of neutron/proton close shell fragment combinations at $T=2$ and 3 $\mathrm{MeV}$. This results ascertain the fact that most favorable fragments have larger phase space than the neighboring nuclei as reported earlier [32, 33].

To this end, it may be mentioned that the differences in the mass distributions or the relative yields calculated using TRMF and FRDM approaches mainly arise due to the differences in the level densities associated with these approaches. The mean values and the fluctuations in the level density parameter and the corresponding level density are even qualitatively different in both the approaches considered. This is possibly stemming from the fact that the single -particle energies in the FRDM based model are temperature independent. The temperature dependence of the excitation energy as required to calculate the level density parameter comes only from the modification of the single-particle occupancy due to the Fermi distribution. In the TRMF approach, the excitation energy for each fragment at a given temperature is calculated self-consistently. Therefore, the deformation and the singleparticle energies changes with temperature.

For the neutron-rich nuclei, the fragments having neutron/proton close shell $N=50,82$ and 100 have maximum possibility of emission at $T=2$ and $3 \mathrm{MeV}$ (for both nuclei ${ }^{250} \mathrm{U}$ and ${ }^{254} \mathrm{Th}$ ). This is a general trend, we could expect for all neutron-rich nuclei. It is worthy to mention some of the recent reports and predictions of multi-fragment fission for neutron-rich uranium and thorium nuclei. When such a neutron-rich nucleus breaks into nearly two fragments, the products exceed the drip-line leaving few nucleons (or light nuclei) free. As a result, these free particles along with the scission neutrons enhance the chain reaction in a thermonuclear device. These additional particles (nucleons or light nuclei) responsible to reach the critical condition much faster than the usual fission for normal thermally fissile nucleus. Thus, the neutron-rich thermally fissile nuclei, which are in the case of ${ }^{246-264} \mathrm{U}$ and ${ }^{244-262} \mathrm{Th}$ will be very useful for energy production.

\section{SUMMARY AND CONCLUSIONS}

The fission mass distributions of $\beta$-stable nuclei ${ }^{236} \mathrm{U}$ and ${ }^{232} \mathrm{Th}$ and the neutron-rich thermally fissile nuclei ${ }^{250} \mathrm{U}$ and ${ }^{254} \mathrm{Th}$ are studied within the statistical theory. The possible combinations are obtained by equating the charge to mass ratio of the parents to that of the fragments. The excitation energies of fragments are evaluated from the temperature dependent self-consistent binding energies at the given $T$ and 
TABLE I: The relative fragmentation yield (R.Y.) $=Y\left(A_{j}, Z_{j}\right)=\frac{P\left(A_{j}, Z_{j}\right)}{\sum P\left(A_{j}, Z_{j}\right)}$ for ${ }^{236} \mathrm{U},{ }^{250} \mathrm{U},{ }^{232}$ Th and ${ }^{254} \mathrm{Th}$, obtained with TRMF at the temperatures $T=1,2$ and $3 \mathrm{MeV}$ are compared with the FRDM prediction (The yield values are normalized to 2).

\begin{tabular}{|c|c|c|c|c|c|c|c|c|c|c|c|}
\hline \multirow{2}{*}{ Parent } & \multirow{2}{*}{$\mathrm{T}(\mathrm{MeV})$} & \multicolumn{2}{|c|}{ TRMF } & \multicolumn{2}{|l|}{ FRDM } & \multirow{2}{*}{ Parent } & \multirow{2}{*}{$\mathrm{T}(\mathrm{MeV})$} & \multicolumn{2}{|l|}{ TRMF } & \multicolumn{2}{|l|}{ FRDM } \\
\hline & & Fragment & R.Y. & Fragment & R.Y. & & & Fragment & R.Y. & Fragment & R.Y. \\
\hline \multirow{11}{*}{${ }^{236} \mathrm{U}$} & \multirow{3}{*}{1} & ${ }^{118} \mathrm{Pd}+{ }^{118} \mathrm{Pd}$ & 0.949 & ${ }^{151} \mathrm{Pr}+{ }^{85} \mathrm{As}$ & 0.210 & \multirow{11}{*}{${ }^{250} \mathrm{U}$} & \multirow{3}{*}{1} & ${ }^{141} \mathrm{Te}+{ }^{109} \mathrm{Zr}$ & 1.454 & ${ }^{160} \mathrm{Pr}+{ }^{90} \mathrm{As}$ & 0.248 \\
\hline & & ${ }^{119} \mathrm{Pd}+{ }^{117} \mathrm{Pd}$ & 0.910 & ${ }^{142} \mathrm{Cs}+{ }^{94} \mathrm{Rb}$ & 0.178 & & & ${ }^{140} \mathrm{Te}+{ }^{110} \mathrm{Zr}$ & 0.491 & ${ }^{161} \mathrm{Pr}+{ }^{89} \mathrm{As}$ & 0.247 \\
\hline & & ${ }^{143} \mathrm{Ba}+{ }^{93} \mathrm{Kr}$ & 0.032 & ${ }^{144} \mathrm{Ba}+{ }^{92} \mathrm{Kr}$ & 0.134 & & & ${ }^{148} \mathrm{Xe}+{ }^{102} \mathrm{Sr}$ & 0.014 & ${ }^{159} \mathrm{Pr}+{ }^{91} \mathrm{As}$ & 0.166 \\
\hline & \multirow{4}{*}{2} & ${ }^{165} \mathrm{Gd}+{ }^{71} \mathrm{Ni}$ & 0.323 & ${ }^{132} \mathrm{Sb}+{ }^{104} \mathrm{Nb}$ & 0.216 & & \multirow{4}{*}{2} & ${ }^{159} \mathrm{Pr}+{ }^{91} \mathrm{As}$ & 0.348 & ${ }^{128} \mathrm{Ag}+{ }^{122} \mathrm{Rh}$ & 0.193 \\
\hline & & ${ }^{164} \mathrm{Gd}+{ }^{72} \mathrm{Ni}$ & 0.264 & ${ }^{133} \mathrm{Te}+{ }^{103} \mathrm{Zr}$ & 0.213 & & & ${ }^{162} \mathrm{Nd}+{ }^{88} \mathrm{Ge}$ & 0.197 & ${ }^{132} \mathrm{In}+{ }^{118} \mathrm{Tc}$ & 0.168 \\
\hline & & ${ }^{163} \mathrm{Gd}+{ }^{73} \mathrm{Ni}$ & 0.0 .221 & ${ }^{151} \mathrm{Pr}+{ }^{85} \mathrm{As}$ & 0.210 & & & ${ }^{160} \mathrm{Pr}+{ }^{90} \mathrm{As}$ & 0.176 & ${ }^{140} \mathrm{Te}+{ }^{110} \mathrm{Zn}$ & 0.140 \\
\hline & & ${ }^{154} \mathrm{Nd}+{ }^{82} \mathrm{Ge}$ & 0.240 & ${ }^{159} \mathrm{Sb}+{ }^{77} \mathrm{Zn}$ & 0.087 & & & ${ }^{173} \mathrm{Gd}+{ }^{77} \mathrm{Ni}$ & 0.175 & ${ }^{141} \mathrm{Te}+{ }^{109} \mathrm{Zn}$ & 0.100 \\
\hline & \multirow{4}{*}{3} & ${ }^{163} \mathrm{Gd}+{ }^{73} \mathrm{Ni}$ & 0.249 & ${ }^{132} \mathrm{Sb}+{ }^{104} \mathrm{Nb}$ & 0.283 & & \multirow{4}{*}{3} & ${ }^{130} \mathrm{Cd}+{ }^{120} \mathrm{Ru}$ & 0.565 & ${ }^{128} \mathrm{Ag}+{ }^{122} \mathrm{Rh}$ & 0.414 \\
\hline & & ${ }^{164} \mathrm{Gd}+{ }^{72} \mathrm{Ni}$ & 0.214 & ${ }^{133} \mathrm{Te}+{ }^{103} \mathrm{Zr}$ & 0.242 & & & ${ }^{132} \mathrm{In}+{ }^{118} \mathrm{Tc}$ & 0.255 & ${ }^{132} \mathrm{In}+{ }^{118} \mathrm{Tc}$ & 0.278 \\
\hline & & ${ }^{136} \mathrm{I}+{ }^{100} \mathrm{Y}$ & 0.143 & ${ }^{134} \mathrm{Te}+{ }^{102} \mathrm{Zr}$ & 0.102 & & & ${ }^{127} \mathrm{Ag}+{ }^{123} \mathrm{Rh}$ & 0.236 & ${ }^{129} \mathrm{Ag}+{ }^{121} \mathrm{Rh}$ & 0.149 \\
\hline & & ${ }^{131} \mathrm{Sb}+{ }^{105} \mathrm{Nb}$ & 0.114 & ${ }^{129} \mathrm{Sn}+{ }^{107} \mathrm{Mo}$ & 0.092 & & & ${ }^{135} \mathrm{Sn}+{ }^{115} \mathrm{Mo}$ & 0.161 & ${ }^{130} \mathrm{Cd}+{ }^{120} \mathrm{Ru}$ & 0.083 \\
\hline \multirow{10}{*}{${ }^{232} \mathrm{Th}$} & \multirow{4}{*}{1} & ${ }^{118} \mathrm{Pd}+{ }^{114} \mathrm{Ru}$ & 0.773 & ${ }^{142} \mathrm{Cs}+{ }^{90} \mathrm{Br}$ & 0.190 & \multirow{10}{*}{${ }^{254} \mathrm{Th}$} & \multirow{4}{*}{1} & ${ }^{142} \mathrm{Sn}+{ }^{112} \mathrm{Zr}$ & 0.439 & ${ }^{145} \mathrm{Sb}+{ }^{109} \mathrm{Y}$ & 0.183 \\
\hline & & ${ }^{140} \mathrm{Xe}+{ }^{92} \mathrm{Kr}$ & 0.515 & ${ }^{144} \mathrm{Ba}+{ }^{88} \mathrm{Se}$ & 0.124 & & & ${ }^{145} \mathrm{Sb}+{ }^{109} \mathrm{Y}$ & 0.291 & ${ }^{163} \mathrm{Ce}+{ }^{91} \mathrm{Ge}$ & 0.118 \\
\hline & & ${ }^{141} \mathrm{Cs}+{ }^{91} \mathrm{Br}$ & 0.174 & ${ }^{120} \mathrm{Ag}+{ }^{112} \mathrm{Tc}$ & 0.123 & & & ${ }^{155} \mathrm{Cs}+{ }^{99} \mathrm{Br}$ & 0.176 & ${ }^{144} \mathrm{Sb}+{ }^{110} \mathrm{Y}$ & 0.115 \\
\hline & & ${ }^{120} \mathrm{Ag}+{ }^{112} \mathrm{Tc}$ & 0.129 & ${ }^{158} \mathrm{Pm}+{ }^{74} \mathrm{Cu}$ & 0.092 & & & ${ }^{157} \mathrm{Ba}+{ }^{97} \mathrm{Se}$ & 0.139 & ${ }^{168} \mathrm{Nd}+{ }^{86} \mathrm{Zn}$ & 0.077 \\
\hline & \multirow{3}{*}{2} & ${ }^{151} \mathrm{Pr}+{ }^{81} \mathrm{Ga}$ & 0.505 & ${ }^{132} \mathrm{Sb}+{ }^{100} \mathrm{Y}$ & 0.213 & & \multirow{3}{*}{2} & ${ }^{176} \mathrm{Sm}+{ }^{78} \mathrm{Ni}$ & 0.370 & ${ }^{144} \mathrm{Sb}+{ }^{110} \mathrm{Y}$ & 0.161 \\
\hline & & ${ }^{132} \mathrm{Sb}+{ }^{100} \mathrm{Y}$ & 0.334 & ${ }^{134} \mathrm{Te}+{ }^{98} \mathrm{Sr}$ & 0.202 & & & ${ }^{175} \mathrm{Sm}+{ }^{79} \mathrm{Ni}$ & 0.290 & ${ }^{178} \mathrm{Eu}+{ }^{76} \mathrm{Co}$ & 0.141 \\
\hline & & ${ }^{166} \mathrm{Gd}+{ }^{66} \mathrm{Fe}$ & 0.134 & ${ }^{129} \mathrm{Sn}+{ }^{103} \mathrm{Zr}$ & 0.146 & & & ${ }^{157} \mathrm{Ba}+{ }^{97} \mathrm{Se}$ & 0.172 & ${ }^{144} \mathrm{Sb}+{ }^{110} \mathrm{Y}$ & 0.132 \\
\hline & \multirow{3}{*}{3} & ${ }^{132} \mathrm{Sb}+{ }^{100} \mathrm{Y}$ & 0.886 & ${ }^{132} \mathrm{Sb}+{ }^{100} \mathrm{Y}$ & 0.252 & & \multirow{3}{*}{3} & ${ }^{127} \mathrm{Rh}+{ }^{127} \mathrm{Rh}$ & 0.803 & ${ }^{127} \mathrm{Rh}+{ }^{127} \mathrm{Rh}$ & 0.325 \\
\hline & & ${ }^{134} \mathrm{Te}+{ }^{98} \mathrm{Sr}$ & 0.148 & ${ }^{129} \mathrm{Sn}+{ }^{103} \mathrm{Zr}$ & 0.207 & & & ${ }^{129} \mathrm{Pd}+{ }^{125} \mathrm{Ru}$ & 0.350 & ${ }^{127} \mathrm{Rh}+{ }^{127} \mathrm{Rh}$ & 0.210 \\
\hline & & ${ }^{155} \mathrm{Nd}+{ }^{77} \mathrm{Zn}$ & 0.063 & ${ }^{134} \mathrm{Te}+{ }^{98} \mathrm{Sr}$ & 0.153 & & & ${ }^{128} \mathrm{Rh}+{ }^{126} \mathrm{Rh}$ & 0.307 & ${ }^{132} \mathrm{Ag}+{ }^{122} \mathrm{Tc}$ & 0.120 \\
\hline
\end{tabular}

the ground state binding energies which are calculated from the relativistic mean field model. The level densities and the yields combinations are manipulated from the convolution integral approach. The fission mass distributions of the aforementioned nuclei are also evaluated from the FRDM formalism for comparison. The level density parameter $a$ and inverse level density parameter $K$ are also studied to see the difference in results with these two methods. Besides fission fragments, the level densities are also discussed in the present paper. For ${ }^{236} \mathrm{U}$ and ${ }^{232} \mathrm{Th}$, the symmetric and nearly symmetric fragmentations are more favorable at temperature $T=$ $1 \mathrm{MeV}$. Interestingly, in most of the cases we find one of the favorable fragment is a close shell or near close shell configuration $(N=82,50$ and $Z=28)$ at temperature $T=2$ and 3 $\mathrm{MeV}$. This result ascertains with our earlier predictions. Further, $\mathrm{Zr}$ isotopes has larger yield values for ${ }^{250} \mathrm{U}$ and ${ }^{254} \mathrm{Th}$ with their accompanied possible fragments at $T=1 \mathrm{MeV}$. The $\mathrm{Ba}$ and $\mathrm{Cs}$ isotopes with their partners are also more possible for ${ }^{254} \mathrm{Th}$. This could be due to the deformed close shell in the region $Z=52-66$ of the periodic table [46]. The $\mathrm{Ni}$ isotopes and the neutron close shell $(N \sim 100)$ nuclei are some of the prominent yields for both ${ }^{250} \mathrm{U}$ and ${ }^{254} \mathrm{Th}$ at temperature $T=2 \mathrm{MeV}$. At $T=3 \mathrm{MeV}$, the neutron close shell $(N=82)$ is one of the largest yield fragments. The symmetric fragmentation ${ }^{127} \mathrm{Rh}+{ }^{127} \mathrm{Rh}$ is possible for ${ }^{254} \mathrm{Th}$ due to the $N=82$ close shell occurs in binary fragmentation. For ${ }^{250} \mathrm{U}$, the larger yield values are confined to the junction of neutron and proton closed shell nuclei.

\section{ACKNOWLEDGMENT}

The author MTS acknowledge that the financial support from UGC-BSR research grant award letter no. F.25-1/201415(BSR)7-307/2010/(BSR) dated 05.11.2015 and IOP, Bhubhaneswar for the warm hospitality and for providing the necessary computer facilities.
[1] D. Vautherin and D. M. Brink, Phys. Lett. B 32, 149 (1970); Phys. Rev. C 5, 626 (1972).

[2] M. K. Pal and A. P. Stamp, Nucl. Phys. A 99, 228 (1967).

[3] S. K. Patra, R. K. Choudhury and L. Satpathy, J. Phys. G. 37,
085103 (2010).

[4] M. Wang, G. Audi, A. H. Wapstra, F. G. Kondev, M. MacCromick, X. Xu and B. Pfeiffer, Chin. Phys. C 36, 1603 (2012).

[5] P. Fong, Phys. Rev. 102, 434 (1956). 
[6] M. Rajasekaran and V. Devanathan, Phys. Rev. C 24, 2606 (1981).

[7] J. D. Walecka, Ann. Phys. 83, 491 (1974).

[8] C. J. Horowitz and B. D. Serot, Nucl. Phys. A 368, 503 (1981).

[9] B. D. Serot and J. D. Walecka, Adv. Nucl. Phys. 16, 1 (1986).

[10] Y. K. Gambhir, P. Ring and A. Thimet, Ann. of Phys. 198, 132 (1990).

[11] S. K. Patra and C. R. Praharaj, Phys. Rev. C 44, 2552 (1991).

[12] P. Arumugam, B. K. Sharma, S. K. Patra and R. K. Gupta, Phys. Rev. C 71, 064308 (2005).

[13] B. K. Sharma, P. Arumugam, S. K. Patra, P. D. Stevenson, R. K. Gupta and W. Greiner, J. Phys. G. 32, L1 (2006).

[14] S. K. Patra, Raj. K. Gupta, B. K. Sharma, P. D. Stevenson and W. Greiner, J. Phys. G. 34, 2073 (2007).

[15] Raj. K. Gupta, S. K. Patra, P. D. Stevenson, C. Beck, and W. Greiner, J. Phys. G. 35, 075106 (2008).

[16] K. Rutz, J. A. Maruhn, P.-G. Reinhard and W. Greiner, Nucl. Phys. A 590, 680 (1995).

[17] M. Balasubramaniam, C. Karthikraj, N. Arunachalam and S. Selvaraj, Phys. Rev. C 90, 054611 (2014); M. Rajasekaran and V. Devanathan, Phys. Rev. C 24, 2606 (1981).

[18] D. W. Bergen and R. R. Fullwood, Nucl. Phys. A 163, 577 (1971).

[19] A. J. Cole, in Fundamental and Applied Nuclear Physics Series - Statistical models for nuclear decay from evaporation to vaporization, edited by R. R. Betts and W. Greiner, Institute of Physics Publsihing, Bristol and Philadelphia, 2000.

[20] J. R. Huizenga and L. G. Moretto, Annu. Rev. Nucl. Sci. 22, 427 (1972).

[21] H. Bethe, Rev. Mod. Phys. 9, 69 (1937).

[22] M.T. Senthil Kannan and M. Balasubramaniam, Eur. Phys. J. A 53, 164 (2017).

[23] P. Möller, J. R. Nix, W. D. Myers and W. J. Swiatecki, At. Data and Nucl. Data Tables 66, 131 (1997).

[24] J. Boguta and A. R. Bodmer, Nucl. Phys. A 292, 413 (1977).
[25] C. E. Price and G. E. Walker, Phys. Rev. C 36, 354 (1987).

[26] P. G. Blunden and M. J. Iqbal, Phys. Lett. B 196, 295 (1987).

[27] P. G. Reinhard, Rep. Prog. Phys. 52, 439 (1989).

[28] S. K. Patra, Phys. Rev. C 48, 1449 (1993).

[29] M. A. Preston and R. K. Bhaduri, Structure of Nucleus, Addison-Wesley Publishing Company, Ch. 8, page 309 (1982).

[30] D. Vautherin, Phys. Rev. C 7, 296 (1973).

[31] J. Dechargé and D. Gogny, Phys. Rev. C 21, 1568 (1980).

[32] M.T. Senthil kannan, Bharat Kumar, M. Balasubramaniam, B. K. Agrawal, S. K. Patra, Phys. Rev. C 95, 064613 (2017).

[33] Bharat Kumar, M.T. Senthil kannan, M. Balasubramaniam, B. K. Agrawal and S. K. Patra, arXiv:1701.00731.

[34] Bharat Kumar, S. K. Biswal, S. K.Singh and S. K. Patra, Phys. Rev. C 92, 054314 (2015).

[35] NIU Yi-Fei, LINAG Hao-Zhao and MENG Jie, Chin. Phys. Lett. 26, 032103 (2009).

[36] B. K. Agrawal, Tapas Sil, J. N. De and S. K. Samaddar, Phys. Rev. C 62, 044307 (2000); ibid 63, 024002 (2001).

[37] B. K. Agrawal, S. K. Samaddar, J. N. De, and S. Shlomo Phys. Rev. C 580, 3004 (1998).

[38] P. Möller, J. R. Nix and K. L. Kratz, At. Data and Nucl. Data Tables 59, 185 (1995).

[39] https://www-nds.iaea.org/RIPL-3/.

[40] B. Nerlo-Pomorska, K. Pomorski, J. Bartel and K. Dietrich, Phys. Rev. C 66, 051302(R) (2002).

[41] J. M. Blatt and V. F. Weisskopf Theoretical Nuclear Physics, Courier Corporation, 1991.

[42] L. Satpathy and S. K. Patra, J. Phys. G. 30, 771 (2004).

[43] Z. Patel et. al., Phys. Rev. Lett. 113, 262502 (2014).

[44] A. Chaudhuri et. al., Phys. Rev. C 91, 044620 (2015).

[45] H. Paca, A. V. Andreev, G. G. Adamian, and N. V. Antonenko, Phys. Rev. C 94, 064614 (2016).

[46] E. F. Jones et. al., J. Phys. G 30, L43 (2004). 